\title{
Transient Evoked Otoacoustic Emissions Testing for Screening of Sensorineural Deafness in Puppies
}

\author{
A. McBrearty and J. Penderis
}

\begin{abstract}
Background: Transient evoked otoacoustic emissions (TEOAE) are widely used for human neonatal deafness screening, but have not been reported for clinical use in dogs.

Hypothesis/Objectives: To investigate the feasibility of TEOAE testing in conscious puppies and the ability of TEOAE testing to correctly identify deaf and hearing ears, as defined by brainstem auditory evoked response (BAER).

Animals: Forty puppies from 10 litters.

Methods: Prospective study on puppies presented for hearing assessment as part of a congenital deafness BAER screening program. Hearing status was determined using BAER. TEOAE testing was performed after the BAER assessment and the results of the TEOAE testing were compared with the hearing status for each ear. Parameters were tested for normality using the D'Agostino Pearson test and comparisons between the deaf and hearing ears were made using Mann-Whitney tests.

Results: TEOAE testing was readily performed in puppies presented for congenital deafness screening. Using analysis parameters based on those used in human neonatal hearing screening, TEOAE testing correctly identified all deaf ears, as defined by BAER testing, with a sensitivity of $100 \%$ (95\% CI: 56-100\%) for diagnosing deafness and specificity of $78 \%$ (95\% CI: $66-87 \%)$.

Conclusions and Clinical Importance: TEOAE testing is an effective screening modality for identifying congenital sensorineural deafness in dogs. In light of the simpler and less expensive equipment, TEOAE testing has the potential to improve access to hearing screening and through this reduce the prevalence of congenital deafness in the dog.
\end{abstract}

Key words: BAER; Canine; Hearing; TEOAE.

C ongenital sensorineural deafness has been reported in over 80 breeds of $\operatorname{dog},{ }^{1}$ and is considered hereditary in most of these breeds. ${ }^{1,2}$ Congenital sensorineural deafness is often, ${ }^{2}$ but not always, ${ }^{3}$ associated with genes conferring white skin pigmentation. Pigment-associated deafness typically presents as total deafness in 1 or both ears. ${ }^{4-9}$ The diagnosis of congenital sensorineural deafness is currently limited to identifying the affected phenotype by auditory screening.

Brainstem auditory evoked response (BAER) testing is the most commonly used test in puppies for deafness screening. ${ }^{2,10}$ For deafness screening, the BAER test is performed with relatively loud sound stimuli and no attempt is made to determine hearing thresholds. ${ }^{5,7,11}$ Puppies with congenital sensorineural deafness have no response to loud stimuli. ${ }^{7,11,12}$ Bilaterally deaf dogs are unable to anticipate dangers such as cars or predators, are difficult to train, might develop anxious or aggressive personalities, and might bite when startled., ${ }^{2,5}$

From the School of Veterinary Medicine, College of Medical, Veterinary and Life Sciences, University of Glasgow, Glasgow, UK. The work was performed at the Small Animal Hospital, College of Medical, Veterinary and Life Sciences, School of Veterinary Medicine, University of Glasgow. Previously presented at 2010 British Small Animal Veterinary Association Annual Congress, Birmingham, UK.

Corresponding author: J. Penderis, School of Veterinary Medicine, College of Medical, Veterinary and Life Sciences, University of Glasgow, Bearsden Road, Glasgow G61 1QH, UK; e-mail: jacques.penderis@glasgow.ac.uk

Submitted April 5, 2011; Revised June 13, 2011; Accepted August 2, 2011.

Copyright $(\mathcal{C} 2011$ by the American College of Veterinary Internal Medicine

$10.1111 / j .1939-1676.2011 .00794 . x$

\begin{tabular}{ll}
\multicolumn{2}{l}{ Abbreviations: } \\
BAER & brainstem auditory evoked response \\
dB & decibel \\
HL & hearing level \\
OAE & otoacoustic emissions \\
SPL & sound pressure level \\
TEOAE & transient evoked otoacoustic emissions
\end{tabular}

Unilaterally deaf dogs have difficulty localizing sound, ${ }^{2,5}$ but otherwise make acceptable pets. Breeding with dogs with unilateral or bilateral congenital sensorineural deafness is associated with an increased risk of deafness in the offspring, and its identification is therefore also important to reduce prevalence of the disease. $^{2,11,13}$ Within the human population, universal hearing screening has been recommended in children by the National Institutes for Health since 1993 (NIH consensus statement, 1993). This largely became feasible after the introduction of the inexpensive and rapid evoked otoacoustic emission tests which have replaced BAER testing as the first choice screening test in many parts of the world.

Otoacoustic emissions (OAEs) are low amplitude sounds produced by the outer hair cells of the cochlea that can be measured by placing a probe containing a microphone in the external ear canal. The 2 forms of OAEs most commonly used for hearing testing are click-evoked transient evoked OAEs (TEOAEs) and distortion product OAEs (DPOAEs). For TEOAEs, broadband click stimuli activate the cochlea simultaneously over a wide frequency region and result in recordable emissions, actively generated by the outer hair cells, with frequencies between 1 and $4 \mathrm{kHz}$ in a normal adult human ear. ${ }^{14}$ TEOAEs can be recorded 
in essentially all normally hearing human ears, ${ }^{15,16}$ and are absent from ears with an audiometric threshold $>20$ to $30 \mathrm{~dB}$ hearing level (HL) ${ }^{14,17}$ TEOAEs have been recorded in anesthetized, normal dogs in 2 small experimental studies, ${ }^{18,19}$ and DPOAEs have been recorded in a study in normal puppies ${ }^{20}$; however, there have been no studies assessing the use of TEOAEs for hearing screening of unsedated puppies. The less expensive and simpler TEOAE equipment has the potential to improve access to hearing screening and through this help to reduce the prevalence of canine deafness.

Due to the pathogenesis of congenital sensorineural deafness in dogs, characterized by complete loss of cochlear outer hair cells, ${ }^{8}$ complete absence of the OAE would be expected in affected dogs. The aim of this study was to therefore to investigate, in unsedated puppies presented for hearing screening, (1) the feasibility of TEOAE testing, (2) the ability of TEOAE testing to identify deaf and hearing ears (as defined by BAER testing), and (3) to analyze the results of the TEOAE tests to improve the chosen protocol for deafness screening.

\section{Materials and Methods}

\section{Animals}

All puppies presenting to the Small Animal Hospital at the University of Glasgow Faculty of Veterinary Medicine for hearing assessment by BAER between June 1, 2009 and January 31, 2010 were eligible for inclusion. Puppies were only included if the owners consented to and sufficient time was available for TEOAE testing to be performed in parallel to the BAER hearing assessment. All puppies were tested without chemical restraint. All puppies were BAER tested but ultimately the time available for TEOAE testing was owner dependent. Informed owner consent was obtained for all included patients and this study was approved by the Faculty of Veterinary Medicine Ethics and Welfare Committee of the University of Glasgow. Age at testing, sex, and breed were recorded for all 40 puppies included.

\section{BAER Screening Testing}

This was performed before TEOAE testing in all cases. BAER testing was performed with 1 of 2 electrodiagnostic machines. ${ }^{\text {a,b }}$ Disposable, 12-mm stainless-steel subdermal needle electrodes were used with the reference electrode rostral to the tragus of the ear being tested, the active electrode at the vertex, and the ground electrode on the dorsal midline of the neck. An alternating polarity, $0.1 \mathrm{~ms}$ duration click stimulus of $80 \mathrm{~dB} \mathrm{nHL}$ was applied at a click rate of 10 per second using headphones held against the opening of the ear canals and a minimum of 512 low noise responses were averaged. ${ }^{\mathrm{c}, \mathrm{d}}$ Low frequency amplifier filter of $100 \mathrm{~Hz}$ and high frequency amplifier filters of $2 \mathrm{~Hz}^{\mathrm{a}}$ and $1 \mathrm{~Hz}^{\mathrm{b}}$ were used. Broadband masking noise was applied simultaneously to the contralateral ear at $30 \mathrm{~dB}$ nHL below the click stimulus to eliminate crossover recordings from a functional contralateral ear. If at least 4 positive peaks were found in response to an $80 \mathrm{~dB}$ nHL click, the ear was considered "hearing"; if not the click stimulus was increased to $100 \mathrm{~dB}$ nHL. Ears with no response at $100 \mathrm{~dB} \mathrm{nHL}$ were considered deaf. BAER results were considered the "gold standard."

\section{TEOAE Testing}

TEOAE testing was performed using commercially available OAE equipment and software installed on a laptop computer. ${ }^{\mathrm{e}, \mathrm{f}}$ Measurements were made using a probe supplied by the same manufacturer and placed in the external ear canal. ${ }^{\mathrm{g}} \mathrm{A}$ disposable rubber probe tip of a suitable size for each ear was selected to form a firm seal within the ear canal. Neither ear cleaning nor inspection of the ear canal was performed before testing. Before testing, probe placement was optimized using the Checkfit function ensuring the temporal waveform was characterized by 2 distinct deflections of opposing polarity and the desired stimulus intensity and a flat stimulus frequency spectrum were achieved. The gain was adjusted to achieve a stimulus as close to $90 \mathrm{~dB}$ sound pressure level (SPL) as possible. The stimuli used were broad-frequency clicks. If the noise after a click exceeded $10 \mathrm{mPa}$ the response to that click was rejected (high noise response). The responses and background noise for a maximum of 260 low noise clicks were amplified and averaged. Half the responses were placed in 1 buffer and half in a 2 nd buffer. Correlation between the 2 buffers gave the whole response reproducibility. The waveform was then fast Fourier transformed and results for the response and noise given for half octave frequency bands centered at 1.0, 1.5, 2.0, 3.0 and $4.0 \mathrm{kHz}$. Data collection stopped after a minimum of 50 low noise clicks had been collected if a pass was achieved in all frequency bands (see below) or when the responses to 260 low noise clicks were collected, whichever occurred first. In ears where no obvious response was recorded, the probe was removed, checked for obstruction of the probe sampling tubes (which were replaced if necessary) and testing was repeated as above. This was performed a maximum of 3 times in each ear.

Data recorded for each run included achieved stimulus intensity, stimulus stability, whole response reproducibility, run duration, and the response and noise in half octave frequency bands centered at $1.0,1.5,2.0,3.0$, and $4.0 \mathrm{kHz}$. The run duration included only the duration of data collection and did not include the time taken to place the probe and assess its optimal placement. To be included in the analysis each run had to meet the following criteria: (1) the achieved peak stimulus intensity had to be between 85 and $95 \mathrm{~dB}$ SPL and (2) stimulus stability had to be $\geq 75 \%$. Runs where these criteria were not achieved failed test validation and were not included in further analysis.

The runs were then analyzed using the following analysis protocol suggested by the manufacturer of the instrument and used for human neonatal hearing screening: The sound-to-noise ratio in each frequency band was calculated (response minus noise). A pass in the frequency bands centered at $1.5,2.0,3.0$, and $4.0 \mathrm{kHz}$ was achieved when the sound-to-noise ratio equaled or exceeded $6 \mathrm{~dB}$ SPL. For the $1 \mathrm{kHz}$ frequency band, a sound-to-noise ratio of $3 \mathrm{~dB}$ SPL or more was considered a pass. For a run to be considered to have passed, passes in at least 3 frequency bands had to be achieved. All other results were considered a fail. The results of the TEOAE test and the BAER test for each ear were compared and the sensitivity and specificity (and 95\% confidence intervals) of the TEOAE test for diagnosing deafness were calculated.

Analysis to optimize the protocol was performed by comparison between the deaf and hearing ears. Parameters investigated included (1) the median sound-to-noise ratios in each frequency band, (2) the percentage of ears achieving a minimum sound-tonoise ratio of $6 \mathrm{~dB}$ SPL ( $3 \mathrm{~dB}$ SPL for the $1 \mathrm{kHz}$ band) in each frequency band, (3) the number of frequency bands achieving a pass for each ear, (4) the number of ears passing 3 or more frequency bands using different minimum sound-to-noise thresholds for the frequency bands $>1 \mathrm{kHz}$, and (5) the whole response 
reproducibility. From these comparisons, a new analysis protocol was developed and tested on this population of ears.

\section{Statistical Analysis}

Parameters were tested for normality using the D'Agostino Pearson test. As the results were not normally distributed, comparisons between the deaf and hearing ears were made using Mann-Whitney tests. All statistical analysis was performed using commercial software except the sensitivity and specificity and $95 \%$ confidence intervals calculations, which were determined using free internet-based software.,

\section{Results}

\section{TEOAE Testing Can Readily Be Performed in} Puppies Presented for Congenital Deafness Screening

Forty puppies (80 ears) from 10 litters were included, comprising 19 Dalmatians, 12 Bull Terriers, and 9 Border Collies. Nineteen puppies were female and 21 were male. The median age was 51 days at the time of testing (range 40-68 days). BAER testing revealed 5 puppies to be unilaterally deaf and 1 to be bilaterally deaf (total of 7 deaf ears). Thirty-seven puppies were bilaterally tested with the TEOAE and 3 puppies were unilaterally tested (total of 77 ears). TEOAE was not attempted in the second ear in 3 puppies because of time constraints dictated by the owner. Two hearing ears (determined by BAER) were excluded from TEOAE analysis because of poor test validation, but TEOAE testing was successfully performed in 75 ears and these ears were included in further analysis (comprising 68 hearing ears and 7 deaf ears). Of the valid results, the median stimulus stability was $99 \%$ (range: $85-100$ ), the peak stimulus intensity was $89.7 \mathrm{~dB}$ SPL (range: $85-93.9$ ), and the median run duration was 73 seconds (range: 23-215).

\section{TEOAE Testing Correctly Identified All Deaf Ears}

Using the predetermined analysis protocol, the TEOAE test correctly identified all deaf ears, resulting in a sensitivity of $100 \%$ (95\% CI: $56-100 \%)$ for diagnosing deafness (Table 1). The specificity of the TEOAE test was $78 \%(95 \%$ CI: $66-87 \%)$. Fifteen ears $(20 \%)$ had discordant results, with the TEOAE test incorrectly classifying these ears as deaf.

Table 1. TEOAE test outcomes in ears defined as hearing or deaf by BAER.

\begin{tabular}{lccc}
\hline & $\begin{array}{c}\text { Hearing } \\
\text { Present } \\
\text { (BAER present) }\end{array}$ & $\begin{array}{c}\text { Deaf } \\
\text { (BAER absent) }\end{array}$ & Total \\
\hline TEOAE pass & 53 & 0 & 53 \\
TEOAE fail & 15 & 7 & 22 \\
Total & 68 & 7 & 75 \\
\hline
\end{tabular}

TEOAE, transient evoked otoacoustic emissions; BAER, brainstem auditory evoked response.

\section{Optimizing the TEOAE Test Analysis Protocol for Deafness Screening}

The sound-to-noise ratio was significantly higher for the hearing ears compared to the deaf ears at the 1.5, 2,3 , and $4 \mathrm{kHz}$ frequency bands $(P=.001, .002, .0004$ and $P<.0001$, respectively). No difference was found in the sound-to-noise ratio for the frequency band centered at $1 \mathrm{kHz}(P=.1)$ (Fig 1$)$. Only $17.6 \%$ hearing ears achieved a pass (sound-to-noise ratio $\geq 3 \mathrm{~dB}$ SPL) in the $1-\mathrm{kHz}$ frequency band and only $36.8 \%$ of hearing ears achieved a pass in the $1.5-\mathrm{kHz}$ frequency band (Fig 2). Seventy-eight percent $(53 / 68)$ hearing ears achieved a pass in 3 or more frequency bands and the deaf ears achieved this threshold in a maximum of 2 bands (Fig 3) suggesting a pass in a minimum of 3 bands is required to prevent deaf ears from being incorrectly classified as hearing. If a pass in 3 or more frequency bands is required but the sound-to-noise ratio threshold for the $1.5 \mathrm{kHz}$ and higher frequency bands is reduced from $6 \mathrm{~dB}$ SPL to $5 \mathrm{~dB}$ SPL, the

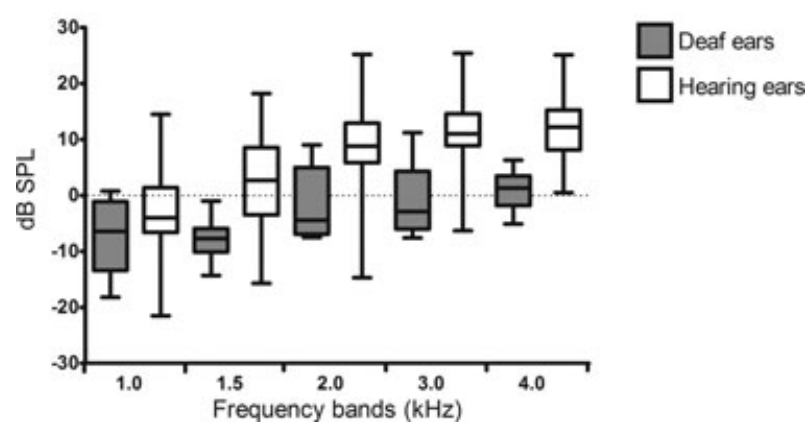

Fig 1. Comparison of the sound-to-noise ratios in deaf and hearing ears at different frequency bands. Significantly higher sound-to-noise ratios are present in hearing ears at all frequency bands, except at the frequency band centered at $1 \mathrm{kHz}(\mathrm{dB}$ $\mathrm{SPL}=$ decibels sound pressure level).

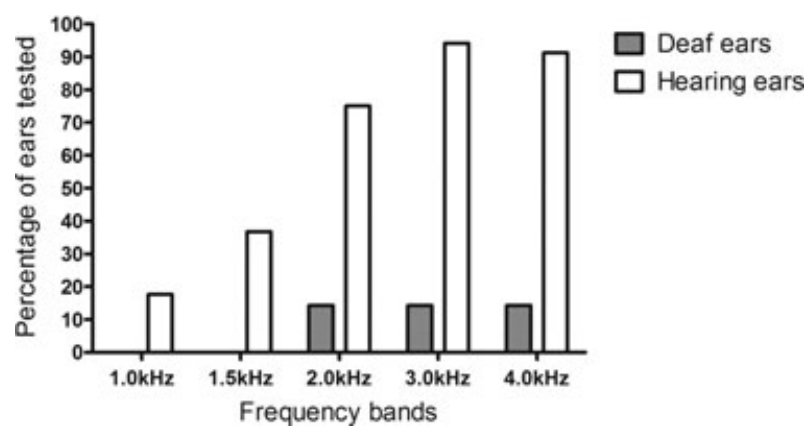

Fig 2. Percentage of ears achieving a pass at different frequency bands based on a sound-to-noise ratio of $\geq 6 \mathrm{~dB}$ SPL $(\geq 3 \mathrm{~dB}$ SPL for the $1 \mathrm{kHz}$ frequency band). The stopping criteria were set to stop the test when a pass was achieved in all frequency bands. However, only a low percentage of hearing ears passed the 1 and the $1.5 \mathrm{kHz}$ frequency bands, despite achieving an overall pass (a pass in at least 3 frequency bands). Adjusting the stopping criteria to stop the test as soon as an overall pass was achieved would have reduced overall test time. 


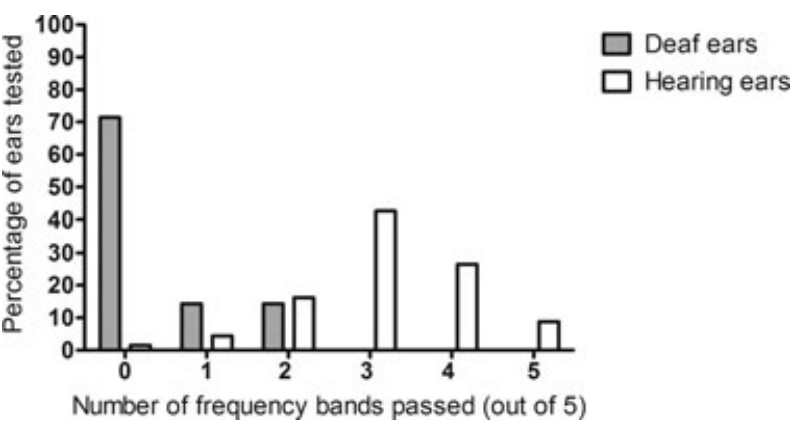

Fig 3. The number of frequency bands passed and failed in individual deaf and hearing ears. An overall pass for an ear was defined as a pass in at least 3 frequency bands. Using the current protocol all deaf ears were correctly identified; however some hearing ears were incorrectly defined as an overall fail. Immediate or delayed retesting of ears defined as a fail would likely have resulted in a reduction in discordant results.

number of discordant results is reduced (with 3 further hearing ears passing) without resulting in any deaf ears passing (Fig 4). Further reduction of the threshold results in some deaf ears passing.

The median whole response reproducibility was significantly different between deaf and hearing ears $(P<.0002)$. The reproducibility threshold with the lowest number of discordant results was approximately $60 \%$, and in this population correctly classified the cochlear status of all but 3 ears, including 1 deaf ear which was classified as hearing (Fig 5). Combining the requirements of a whole response reproducibility of $>60 \%$ and a pass in 2 or more frequency bands (with a pass $\geq 3 \mathrm{~dB}$ SPL for $1 \mathrm{kHz}$ band and $\geq 5 \mathrm{~dB}$ SPL for all other frequency bands) resulted in discordant results in only 5 ears; however these parameters did incorrectly classify 1 deaf ear as having acceptable cochlear function.

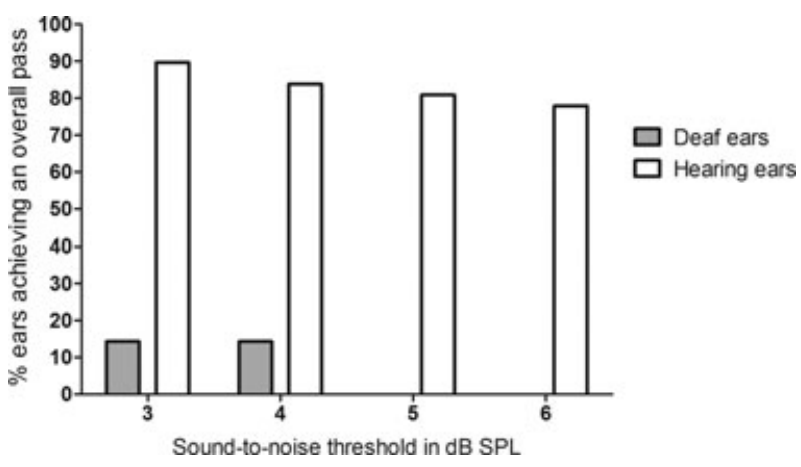

Fig 4. The effect of reducing the sound-to-noise thresholds for frequency bands centered at $1.5 \mathrm{kHz}$ and above. Reduction of the sound-to-noise threshold from 6 to $5 \mathrm{~dB}$ SPL increased the number of hearing ears correctly identified without incorrectly classifying deaf ears. Reduction of the sound-to-noise threshold below $5 \mathrm{~dB}$ SPL resulted in some deaf ears incorrectly being classified as having adequate cochlear function. The sound-to-noise threshold for the $1 \mathrm{kHz}$ frequency band was unchanged at $\geq 3 \mathrm{~dB}$ SPL.

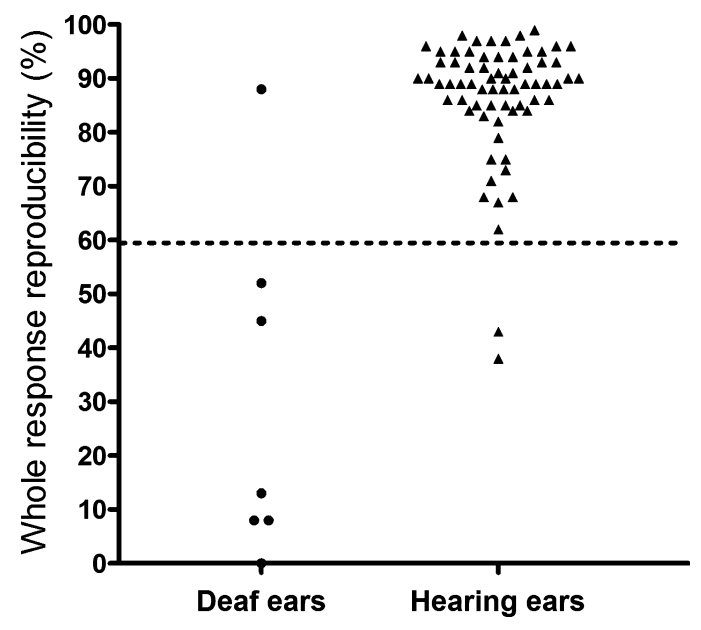

Fig 5. The whole response reproducibility for each ear tested by the TEOAE test. Each symbol ( $\boldsymbol{\Delta}$ or $\bullet$ ) represents an individual ear. The whole response reproducibility was significantly different between deaf and hearing ears $(P<.0002)$. Whole response reproducibility may be used to discriminate between deaf and hearing ears, with higher whole response reproducibility in ears with normal cochlear function. The reproducibility threshold with the lowest number of discordant results was approximately $60 \%$, and in this population correctly classified the cochlear status of all but 3 ears.

\section{Discussion}

This study demonstrates the utility of TEOAE as a screening modality for congenital sensorineural deafness in puppies. The findings of this study demonstrate that the TEOAE test performed reasonably well within the clinical setting, correctly identifying all the deaf ears using the predetermined test protocol in this prospective study. TEOAE equipment is widely available and easier to use than the equipment required for BAER and does not require insertion of subcutaneous needle electrodes. The test protocol used did incorrectly classify some hearing ears as having abnormal cochlear function. The comparative situation in human neonatal screening is to use the TEOAE test as a first screening modality with those patients that fail the initial assessment being retested. ${ }^{21}$ If patients still fail then they are referred for further hearing assessment. ${ }^{21}$ Within the veterinary setting, establishment of testing centers using TEOAE in private practices would result in greater access to deafness screening, allowing improved selection of breeding animals. Puppies that fail can be retested at a later date and those that consistently fail can then be referred to a regional test centre for BAER assessment, particularly if these puppies are identified as bilaterally deaf on TEOAE testing or the owner would like to use them as breeding animals. Excluding dogs with congenital sensorineural deafness from breeding programs reduces the incidence of deafness in subsequent litters ${ }^{13}$ and better availability of deafness screening in dogs would have a positive effect on animal welfare.

TEOAE testing demonstrated significant differences in the sound-to-noise ratios between deaf and hearing ears in 4 of the 5 frequency bands assessed. The major- 
ity $(80 \%)$ of deaf and hearing ears were correctly identified with the chosen analysis protocol but the confidence intervals for the sensitivity and specificity of the test were wide, possibly reflecting the relatively small number of ears included in the study. Fifteen hearing ears were incorrectly classified as deaf by the TEOAE test. Possible reasons for the discordance between the TEOAE and BAER results are that the TEOAE test is more affected by ambient noise and movement of puppies, presence of outer or middle ear pathology (this will affect the TEOAE test more), that TEOAE analysis criteria were overly stringent, or that in discordant cases deafness was caused by pathology of the inner hair cells or auditory nerve with preservation of the outer hair cells (although these pathologies are thought to be rare in dogs). One difference between the TEOAE and BAER tests is that the TEOAE records the cochlear response at various frequency bands, whereas the BAER records the electrical activity in the cochlea, cochlear nerve and brainstem in response to a broadband stimulus. The requirement to achieve threshold sound-to-noise amplitudes in several different frequency bands is a more stringent test of cochlear function than the BAER. ${ }^{16}$

The analysis protocol in this study was based on the neonatal hearing screening protocol recommended by the equipment manufacturer, as there is little consensus on the exact pass criteria in human audiology. ${ }^{16}$ The identification of 15 hearing ears as deaf, but no deaf ears as hearing, suggested that this protocol may be overly stringent. As puppies are presented for hearing testing as a litter, the presence of a consistent fail in 1 ear of 1 puppy will likely result in the breeder being required to travel to a regional test center (albeit with just the puppies failing the initial screening). Comparisons between the results for deaf and hearing ears were made to enable alteration of the analysis protocol to try and reduce the number of discordant results. No difference in the sound-to-noise ratio was found between deaf and hearing ears for the $1 \mathrm{kHz}$ frequency band in this study and $<20 \%$ of hearing ears passed this frequency band. However no deaf ears passed at this frequency. Removing this frequency band from the analysis protocol would not improve the discrimination of deaf and hearing ears and because data are collected simultaneously from all frequency bands would not reduce test time. Retrospective analysis of the data showed that the number of frequency bands required for an overall pass cannot be reduced from 3 to 2 as 1 deaf ear achieved a pass in 2 frequency bands. Reducing the sound-to-noise ratio threshold from $6 \mathrm{~dB}$ SPL to $5 \mathrm{~dB}$ SPL for all frequency bands above the $1 \mathrm{kHz}$ band resulted in 3 additional hearing ears passing without any deaf ears passing. The whole response reproducibility, which is a measure of the repeatability of the response to the clicks, discriminated well between deaf and hearing ears when a threshold of $60 \%$ was used. Whole response reproducibility is included in the analysis protocol in some human neonatal screening programs. $^{22-25}$
Using these findings, a revised protocol was designed in which an overall pass was defined as a pass (sound-to-noise ratio of $\geq 3 \mathrm{~dB}$ SPL for the $1 \mathrm{kHz}$ frequency band and a sound-to-noise ratio $\geq 5 \mathrm{~dB}$ SPL for the frequency bands $>1 \mathrm{kHz}$ ) in at least 2 frequency bands and a whole response reproducibility of $>60 \%$. In this population, the number of discordant results was substantially reduced; however, these revised test parameters did incorrectly classify 1 deaf ear as having normal cochlear function and this is unlikely to be acceptable.

Reducing test time is important as it will improve client compliance, increase the likelihood of successful testing in all ears, and increase the number of ears that can be assessed in a given time. The median run duration in this study was 73 seconds (range: 23-215). The stopping criteria were deliberately chosen to be more stringent than the pass criteria to maximize the data collected from each ear. A pass in all 5 frequency bands (which results in test termination) was achieved in only 6 ears and the test time for many ears could have been reduced if testing was terminated as soon as the pass criteria (ie, 3 passes out of 5) were reached. Sleeping puppies were quicker to test than active puppies as fitting the probe was easier, stimulus stability was improved, and there was less ambient noise. In children, refinement of the analysis protocols and stopping criteria means that in some circumstances a response adequate for a pass can now be detected in as little as 7 seconds. ${ }^{j}$ The findings of this study suggest that the protocol could be improved by changing the stopping criteria to stop as soon as an overall pass is achieved and reducing the threshold for a pass in the frequency bands centered at $1.5 \mathrm{kHz}$ and above from 6 to $5 \mathrm{~dB}$ SPL. However, these improvements would require validation in a prospective study. It must be accepted that either this or the original analysis protocol used in this study will generate a number of hearing ears identified as deaf, requiring immediate or delayed retesting and euthanasia of puppies on the basis of the results of the TEOAE test as used in this study would not be appropriate.

This study was limited by the fact that BAER was used as the gold standard for the diagnosis of congenital sensorineural deafness. While BAER is widely accepted as the best test currently available for congenital sensorineural deafness testing in puppies, it is not specific for this form of auditory dysfunction. Other forms of deafness might also result in abolishment of the BAER, although these were less likely due to the young age of the dogs included in this study. Otoscopy was not performed on the puppies in this study because it would require increased test time, would stimulate the puppies, external ear canal pathology is unusual at this age and it is not routinely performed in human neonates before TEOAE testing. ${ }^{16}$ Dogs show BAER to sound stimuli to frequencies as high as $32 \mathrm{kHz}^{26}$; however, despite using a click stimulus, the TEOAE response is only measured between 0.5 and $4 \mathrm{kHz}$. As dogs with congenital sensorineural deafness typically have complete deafness in 
the affected ear, ${ }^{4-7}$ it would be expected that responses would be affected in the frequency region tested. The identification of all deaf ears by the TEOAE test in this study, also suggests this is the case.

Despite these limitations, this study demonstrated that TEOAE testing is feasible in puppies presented for congenital sensorineural deafness and all deaf ears were correctly identified. A suggested analysis protocol is described to discriminate between hearing and deaf ears for congenital deafness screening and is a starting point for further studies. In light of the ease, speed, and lower cost of the equipment, TEOAE testing has the potential to improve access to hearing screening and through this help to reduce the prevalence of canine deafness.

\section{Footnotes}

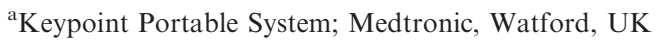

bynergy N-EP 10-channel System; Medelec, CareFusion, San

Diego, CA

${ }^{\mathrm{c}}$ Model 31E25; Medtronic

${ }^{\mathrm{d}}$ Model TDH49P; Medelec

${ }^{\mathrm{e}}$ Echoport ILO-288 USB II; Otodynamics Ltd, Hatfield, UK

${ }^{\mathrm{f}}$ EZ-Screen 2 Software; Otodynamics Ltd

${ }^{\mathrm{g}}$ UGD TE+DPOAE Probe; Otodynamics Ltd

${ }^{\mathrm{h}}$ GraphPad Prism v5 software; GraphPad Software, Inc, La Jolla, CA

${ }^{\mathrm{i}}$ VassarStats Software (http://faculty.vassar.edu/lowry/VassarStats.html)

${ }^{\mathrm{j}}$ http://www.otodynamics.com

\section{Acknowledgments}

This study was supported by funding from Kennel Club Charitable Trust and Bull Terrier Clinical Studies Fund.

\section{References}

1. Strain GM. Deafness prevalence and pigmentation and gender associations in dog breeds at risk. Vet $\mathbf{J}$ 2004;167:23-32.

2. Strain GM. Congenital deafness and its recognition. Vet Clin North Am Small Anim Pract 1999;29:895-907.

3. Wilkes MK, Palmer AC. Congenital deafness and vestibular deficit in the Dobermann. J Small Anim Practice 1992;33:218-224.

4. Igarashi M, Alford BR, Cohn AM et al. Inner ear anomalies in dogs. Ann Otol Rhinol Laryngol 1972;81:249-255.

5. Strain GM. Aetiology, prevalence and diagnosis of deafness in dogs and cats. Br Vet J 1996;152:17-36.

6. Ahlstrom LA, Wilson WJ, Mills PC. Unilateral deafness in a white Bull Terrier diagnosed by BAER assessment. Aust Vet $\mathbf{J}$ 2005;83:742-743.
7. Strain GM, Clark LA, Wahl JM et al. Prevalence of deafness in dogs heterozygous or homozygous for the merle allele. $\mathrm{J}$ Vet Intern Med 2009;23:282-286.

8. Lurie MH. The membranous labyrinth in the congenitally deaf Collie and Dalmatian dog. Laryngoscope 1948;58:279-287.

9. Branis $\mathbf{M}$, Burda H. Inner ear structure in the deaf and normally hearing Dalmatian dog. J Comp Pathol 1985;95:295299.

10. Wilson WJ, Mills PC. Brainstem auditory-evoked response in dogs. Am J Vet Res 2005;66:2177-2187.

11. Platt S, Freeman J, di Stefani A et al. Prevalence of unilateral and bilateral deafness in Border Collies and association with phenotype. J Vet Intern Med 2006;20:1355-1362.

12. Famula TR, Oberbauer AM, Sousa CA. A threshold model analysis of deafness in Dalmatians. Mamm Genome 1996;7:650-653.

13. Wood JL, Lakhani KH. Prevalence and prevention of deafness in the Dalmatian - assessing the effect of parental hearing status and gender using ordinary logistic and generalized random litter effect models. Vet J 1997;154:121-133.

14. Kemp DT. Otoacoustic emissions, their origin in cochlear function, and use. Br Med Bull 2002;63:223-241.

15. Lonsbury-Martin BL, Martin GK. Otoacoustic emissions: basic studies in mammalian models. In: Manley GA, Fay FF, Popper AN, eds. Active Processes and Otoacoustic Emissions, 1st ed. New York: Springer Science and Business Media; 2008:261-303.

16. Hall JW. Handbook of Otoacoustic Emissions. San Diego, CA: Singular Publishing Group; 2000.

17. Lonsbury-Martin BL, Martin GK. The clinical utility of distortion-product otoacoustic emissions. Ear Hear 1990;11:144 154.

18. Sockalingam R, Filippich L, Sommerlad S et al. Transient-evoked and 2F1-F2 distortion product oto-acoustic emissions in dogs: preliminary findings. Audiol Neurootol 1998;3:373-385.

19. Sims MH, Rogers RK, Thelin JW. Transiently evoked otoacoustic emissions in dogs. Prog Vet Neurol 1994;5:49-56.

20. Schemera B, Blumsack JT, Cellino AF et al. Evaluation of otoacoustic emissions in clinically normal alert puppies. Am J Vet Res 2011;72:295-301.

21. Maxon AB, White KR, Behrens TR et al. Referral rates and cost efficiency in a universal newborn hearing screening program using transient evoked otoacoustic emissions. J Am Acad Audiol 1995;6:271-277.

22. De Capua B, Costantini D, Martufi C et al. Universal neonatal hearing screening: the Siena (Italy) experience on 19,700 newborns. Early Hum Dev 2007;83:601-606.

23. Korres S, Nikolopoulos TP, Peraki EE et al. Outcomes and efficacy of newborn hearing screening: Strengths and weaknesses (success or failure?). Laryngoscope 2008;118:1253-1256.

24. Doyle KJ, Burggraaff B, Fujikawa S et al. Newborn hearing screening by otoacoustic emissions and automated auditory brainstem response. Int J Pediatr Otorhinolaryngol 1997;41:111-119.

25. Rhodes MC, Margolis RH, Hirsch JE et al. Hearing screening in the newborn intensive care nursery: Comparison of methods. Otolaryngol Head Neck Surg 1999;120:799-808.

26. Ter Haar G, Venker-van Haagen AJ, de Groot HN et al. Click and low-, middle-, and high-frequency toneburst stimulation of the canine cochlea. J Vet Intern Med 2002;16:274-280. 